\title{
Los procesos de reestructuración socialmente sostenibles en el Impuesto sobre Sociedades ${ }^{1}$
}

\author{
Marina Aguilar Rubio \\ Profesora Derecho Financiero y Tributario de la Universidad de Almería
}

DOI: $10.1387 /$ reves. 15261

Fecha de entrada: 30/01/2015

Fecha de aceptación: 10/04/2015

\begin{abstract}
Sumario: I. Introducción. II. La transformación de sociedades capitalistas en sociedades cooperativas: Reestructuraciones socialmente sostenibles. II.1. Las reestructuraciones socialmente responsables en la Unión Europea. II.2. Las reestructuraciones socialmente sostenibles en España. III. Aproximación a la fiscalidad de la transformación de la sociedad de capital en cooperativa. III.1. Aspectos fiscales reestructuración empresarial: precisión terminológica. III.2. La transformación de la sociedad de capital en sociedad cooperativa en el Impuesto sobre Sociedades. IV. Notas conclusivas. V. Bibliografía.
\end{abstract}

\begin{abstract}
Resumen:
Este trabajo se centra en los procesos de reestructuración societaria que implican la transformación de una sociedad de capital en una sociedad cooperativa porque los trabajadores de la misma se convierten en socios de la cooperativa resultante de la operación. En términos generales, dos son los escenarios principales en los que se lleva a cabo una transformación de estas características: en primer lugar, por ausencia de relevo generacional en la empresa y, en segundo lugar, por la toma del control societario por los empleados de la empresa cuando esta se encuentra en situación de crisis.

Desde la perspectiva fiscal, la transformación de una sociedad de capital en una sociedad cooperativa supone un cambio de régimen tributario aplicable como sujetos del Impuesto sobre Sociedades los que se pone de manifiesto, entre otras, en cuestiones de tanto calado como la interrupción anticipada del periodo impositivo de la entidad originaria; el cambio de tipo de gravamen aplicable; o la manera de aplicar la compensación de pérdidas a que se tenga derecho.
\end{abstract}

1 Estudio realizado en el marco del Proyecto de Investigación del Ministerio de Economía y Competitividad: «Integración cooperativa y reestructuraciones socialmente responsables. Mecanismo de creación e empleo e incremento de productividad empresarial» (DER2013-48864C2-1-P). 


\title{
Palabras clave:
}

Cooperativas, Impuesto sobre Sociedades, reestructuraciones socialmente sostenibles, transformación.

\begin{abstract}
:
The work focuses on corporate restructuring processes involving the transformation of a capital company in a cooperative society because workers become members of the cooperative resulting from the operation. There are two main scenarios in which takes place a transformation of these characteristics: first, by the absence of generational replacement in the company and, secondly, by employees taking control of the corporate company when this is in crisis.

From a tax perspective, the transformation of a capital company into a cooperative generates evident changes of tax regime in corporation tax in such depth issues as early termination of the taxation period of the parent entity; applicable tax rate; or how to apply the compensation of losses to which it is entitled, among others.
\end{abstract}

\section{Keywords:}

cooperative, corporation tax, transformation, socially sustainable restructuring.

\section{Claves ECONLIT}

K290 y K340

\section{Introducción}

El concepto mercantil de modificación estructural de una sociedad incluye dentro de su ámbito las operaciones de transformación, fusión, de escisión, de cesión global de activo y pasivo y de traslado internacional de del domicilio fiscal de cualquier sociedad que tenga la consideración de mercantil, bien por la naturaleza de su objeto, bien por la forma de su constitución. ${ }^{2}$ En ningún caso pretendemos aquí abordar todos estos fenómenos, ni siquiera todas las posibilidades que ofrece la transformación empresarial. Nuestro trabajo se va a centrar exclusivamente en estudiar un proceso concreto de reestructuración societaria que implica la transformación de una sociedad normalmente limitada o anónima en una sociedad cooperativa por mor de su adquisición por los trabajadores de la misma. Un fenómeno

2 Arts. 1 y 2.1 Ley 3/2009, de 3 de abril, de Modificaciones Estructurales de las Sociedades Mercantiles. 
conocido como recuperación de empresas o reconversión de empresas pero que nosotros preferimos llamar reestructuraciones socialmente sostenibles. ${ }^{3}$

El art. 7 de la Ley 3/2009, de 3 de abril, de Modificaciones Estructurales de las Sociedades Mercantiles (LMESM) regula tanto la transformación de una cooperativa en otro tipo social como el supuesto inverso, de otro tipo social en cooperativa remitiéndose, en lo referente a los requisitos y efectos de la transformación de la sociedad cooperativa a la legislación que le sea aplicable. ${ }^{4} \mathrm{El}$ art. 69 de la Ley 27/1999, de 16 de julio, General de Cooperativas (LGC) establece que cualquier sociedad que no tenga carácter cooperativo puede transformarse en una sociedad cooperativa. Y las Leyes autonómicas hacen lo propio. ${ }^{5}$

3 El Dictamen sobre el tema «Cooperativas y reestructuración» del Comité Económico y Social Europeo, de 25 de abril de 2012 (CESE1049-2012), acuńa el término de «reestructuraciones socialmente responsables» (p. 5) pero nosotros —y no somos los únicos- preferimos el concepto de reestructuraciones socialmente sostenibles que, creemos, refleja mejor los fines perseguidos en estos casos, puesto que la recuperación de empresas por parte de los trabajadores persigue fundamentalmente la sostenibilidad de la actividad empresarial y de los empleos que de ella dependen.

Este término tiene una significación más amplia, puesto que cabría en el también la transformación de la sociedad de capital en sociedad laboral, pero nosotros lo vamos a utilizar en este trabajo en referencia exclusivamente a las operaciones de transformación de una sociedad de capital en una cooperativa de trabajo asociado.

4 También admite la transformación de sociedades cooperativas en sociedades cooperativas europeas y viceversa, disponiendo su regulación por el Reglamento $1435 / 2003$ y por las normas que lo desarrollen.

5 Las Leyes autonómicas regulan la transformación en los artículos que detallamos en la siguiente tabla:

\begin{tabular}{|c|c|c|}
\hline & $\begin{array}{l}\text { Transformación } \\
\text { de la cooperativa }\end{array}$ & $\begin{array}{l}\text { Transformación } \\
\text { en cooperativa }\end{array}$ \\
\hline Ley 14/2011 de Sociedades Cooperativas Andaluzas* & Art. 78.1 a 4 & Art. 78.5 \\
\hline Ley 9/1998 de Cooperativas de Aragón & Art. 66 & \\
\hline Ley 6/2013 de Cooperativas de Cantabria & Art. 89 & Art. 90 \\
\hline Ley 18/2002 de Cooperativas de Cataluña & Art. 84 & Art. 85 \\
\hline Ley 4/2002 de Cooperativas de Castilla y León & Art. 99 & Art. 86 \\
\hline Ley $11 / 2010$ de Cooperativas de Castilla La Mancha & Art. 100 & Art. 99 \\
\hline Ley 4/1999 de Cooperativas de la C. de Madrid & Arts. 83 a 90 y 92 & Art. 91 \\
\hline Ley $8 / 2003$ de Cooperativas de la C. Valenciana & Art. 79 & Art. 80 \\
\hline Ley 2/1998 de Sociedades Cooperativas Extremadura & Arts. 74 a 80 & Art. 81 \\
\hline Ley 5/1998 de Cooperativas de Galicia & Art. 84 & Art. 85 \\
\hline Ley $1 / 2003$ de Cooperativas de Islas Baleares & \multicolumn{2}{|c|}{ No regula la transformación } \\
\hline Ley 8/2006 de Cooperativas de la Región de Murcia & Art. 94 & Art. 95 \\
\hline Ley 4/2010 de Cooperativas del Principado de Asturias & Art. 115 & Art. 116 \\
\hline Ley 4/1993 de Cooperativas del País Vasco & Art. 85 & Art. 86 \\
\hline Ley 4/2001 de Cooperativas de La Rioja & Art. 115 & Art. 116 \\
\hline Ley Foral 12/2006 de Cooperativas de Navarra & Art. 59 & Art. 59.2 \\
\hline
\end{tabular}

* Esta Ley está desarrollada por el Decreto 123/2014 por el que se aprueba el Reglamento de la Ley 14/2011, de 23 de diciembre, de Sociedades Cooperativas Andaluzas. Los artículos 65 y 66 de esta norma regulan el procedimiento de transformación de sociedades cooperativas y el procedimiento de transformación y en sociedades cooperativas respectivamente. 
En términos generales, dos son los escenarios principales en los que se lleva a cabo una transformación de estas características: en primer lugar, que llegado el momento del cese de la actividad empresarial no haya un sucesor dispuesto a hacerse cargo de la empresa, en cuyo caso, el propietario puede entregarla a los empleados, quienes la convertirán en una cooperativa de trabajo asociado. Y en segundo lugar, que los empleados adquieran empresas en crisis, esto es, empresas en riesgo de liquidación o de concurso de acreedores, para convertirlas en una cooperativa de trabajadores.

\section{La transformación de sociedades capitalistas en sociedades cooperativas: Reestructuraciones socialmente sostenibles}

\section{II.1. Las reestructuraciones socialmente responsables en la Unión Europea}

Cuando se habla de empresas reconvertidas, lo primero que suele venir a la mente son las escenas de ocupación de fábricas en Argentina por parte de los trabajadores de las mismas con protestas y manifestaciones y de desalojos violentos llevados a cabo por la policía. No obstante, en los Estados miembros de la Unión Europea se han producido numerosas reconversiones de empresas, ${ }^{6}$ como consecuencia de la crisis económica y de la deslocalización empresarial que persigue, fundamentalmente, abaratar los costes laborales. ${ }^{7}$

La Comisión Europea señala que «las empresas que consiguen reestructurarse de manera socialmente responsable suelen ser las que mejor historial tienen en cuanto a competitividad y adaptación en el mercado». ${ }^{8}$ Las cooperativas están demostrando una mayor resistencia en tiempos de crisis

${ }^{6}$ Un informe elaborado por CECOP-CICOPA Europe (Confederación europea de cooperativas y empresas propiedad de sus trabajadores activas en los sectores industrial y de servicios) (2013) Business Transfers to Employees under the Form of a Cooperative in Europe. Opportunities and Challenges, , p. 3, sitúa a España como uno de los países de la UE donde esta práctica está más extendida, junto con Francia e Italia. La CECOP también ha registrado desde 2009 los efectos de la crisis en las empresas de su red. Las cooperativas de los países que cuentan con una mayor implantación y experiencia de este sector (a la sazón, Francia, Italia, Espańa) parecen ser más resistentes al deterioro de la crisis que las empresas convencionales activas en los mismos sectores y territorios.

7 Europa también estudia de cerca los procesos de reconversión de empresas ante la amenaza del cierre de plantas multinacionales que trasladan su producción en busca de mano de obra más barata. En este contexto, el proyecto This is not Detroit, surgido a raíz del cierre anunciado de la planta Opel en Bochum (45.000 puestos de trabajo en Alemania, Cuenca del Rühr), invita a la reflexión sobre el tipo de industria que se plantea en el futuro.

${ }^{8} \operatorname{COM}(2005) 120$ final. Reestructuraciones y empleo-Anticipar y acompañar las reestructuraciones para desarrollar el empleo: el papel de la Unión Europea, p. 11. 
que las empresas convencionales. Ello se pone de manifiesto de forma especialmente relevante en el caso de las cooperativas financieras, las cooperativas de trabajo asociado en la industria y los servicios, las cooperativas sociales y las cooperativas formadas por PYMES. ${ }^{9}$ En España, donde la crisis ha sido especialmente dura, la reducción del empleo en 2008 y 2009 fue del 4,5\% en el sector cooperativo, mientras que alcanzó el 8\% en las empresas convencionales. $\mathrm{Y}$ en 2010 las cooperativas de trabajo asociado incrementaron el número de puestos de trabajo en un $0,2 \%$ mientras que el empleo total disminuyó en las empresas convencionales en un $3,2 \% .{ }^{10}$

Las cooperativas están adquiriendo cada vez más importancia en la Unión Europea, donde existen alrededor de 160.000 empresas cooperativas, que pertenecen a 123 millones de miembros y proporcionan empleo a 5,4 millones de personas (incluidas unas 50.000 cooperativas en los sectores de la industria y los servicios en las que trabajan 1,4 millones de personas), y contribuyen aproximadamente en un 5\%, de media al PIB de los Estados miembros. ${ }^{11}$ Asimismo, durante los últimos años, se han creado cientos de cooperativas industriales y de servicios debido a la reestructuración de empresas en crisis o sin sucesor, salvando y revitalizando así actividades económicas y puestos de trabajo a escala local y los grupos de cooperativas industriales y de servicios han influido de forma notable en el desarrollo regional de algunas de las regiones más industrializadas de la Unión Europea. Las cooperativas «sociales» especializadas en la integración laboral proporcionan empleo a más de 30.000 personas discapacitadas o desfavorecidas en los sectores de la industria y los servicios. A su vez, las cooperativas se han convertido en un modelo para los trabajadores autónomos y las profesiones liberales y han adquirido notable importancia en nuevos sectores. ${ }^{12}$ Ello deja constancia del importante papel que desem-

9 Según el informe de la OIT, elaborado por Birchall, J. (20I4): Resilience of the cooperative business model in times of crisis, Ginebra, las cooperativas financieras se mantienen sólidas desde el punto de vista financiero; las cooperativas de consumidores presentan un aumento del volumen de negocios; las cooperativas de trabajo asociado registran un incremento, dado que la población elige la forma de cooperativa para responder a las nuevas situaciones económicas (Versión en español: Resiliencia del modelo de negocio de las cooperativas en tiempos de crisis, 2014, passim. disponible en http://www.ilo.org/wcmsp5/groups/public/---edemp/documents/ publication/wcms235766.pdf.)

10 CESE1049-2012 (2012): Dictamen sobre el tema "Cooperativas y reestructuración", p. 9.

11 PE 2012/2321(INI)) (2013): Informe sobre la contribución de las cooperativas a la salida de la crisis, p. 4.

12 En referencia a actividades como los servicios sociales o de atención sanitaria, los servicios digitales y de apoyo empresarial y los servicios de interés general prestados anteriormente por el sector público (por ejemplo, servicios medioambientales y gestión de espacios naturales, educación y cultura, y producción de energía a partir de fuentes renovables.) 
peñan las cooperativas en la Unión Europea en términos económicos, sociales, laborales y de desarrollo sostenible. ${ }^{13}$

En este contexto, las transmisiones de empresas a los empleados mediante la creación de una cooperativa y otras formas de propiedad por parte de los trabajadores podría ser la mejor forma de garantizar la continuidad de la empresa. Por tanto, el Parlamento Europeo - haciéndose eco de que el recurso a la adquisición por parte de los trabajadores se está convirtiendo en una práctica cada vez más difundida en los Estados miembros en respuesta a la crisis económica- ha abogado por respaldar este tipo de operaciones mediante una partida específica en el presupuesto de la UE que también incluya instrumentos financieros y ha solicitado que se cree un mecanismo europeo diseñado para fomentar la creación de cooperativas y, en particular, las transmisiones empresariales en forma de cooperativa, mediante, por ejemplo, los fondos de mutualización con la participación del Banco Europeo de Inversiones, los interlocutores sociales y las partes interesadas del movimiento cooperativo. ${ }^{14}$

\section{II.2. Las reestructuraciones socialmente sostenibles en España}

En España hay casi un centenar de empresas reconvertidas en cooperativa, y la mayoría se han constituido en los últimos años, al albur de la crisis económica ${ }^{15}$ y existen tantas experiencias distintas como empresas reconvertidas en cooperativa. ${ }^{16}$

Muchas veces los conflictos se resuelven favorablemente si cuentan con el apoyo de instituciones públicas, como es el caso de la cooperativa barcelonesa Mec2010, las metalúrgicas navarras Tafinox y Curvados Alzania

13 Este rol, junto con el reconocimiento a su contribución al logro del objetivo de desarrollo económico y social sostenible de las comunidades regionales y locales se han visto reforzados por la Estrategia Europa 2020 y por el programa Horizonte 2020.

14 PE 2012/2321(INI)) (2013, p. 25).

15 Según datos de 2012 de la Confederación Española de Cooperativas de Trabajo Asociado (COCETA). Pero puede que haya más dado que muchas de las empresas recuperadas no están inscritas en las federaciones y no hay manera de saber si provienen de la quiebra de una empresa mercantil o de un ERE. O los nuevos dueños, sus trabajadores, no quieren que se sepa que la empresa ahora es propiedad de los trabajadores, por miedo a perder clientela.

16 Por lo general, los conflictos como los argentinos son en Espańa cooperativas históricas, creadas hace más de 30 ańos, como el caso de Gramagraf o Mol Matric, en Barcelona (VILNitzky, M. y Ruiz, O. (2010): «Dossier Empresas recuperadas», El Viejo topo, núm. 270-271, julio-agosto 2010, passim.) 
o Muebles Viana, cuyos trabajadores lograron quedarse con la maquinaria y avanzar en la recuperación. ${ }^{17}$

La formación de este tipo de cooperativas en España también proviene de algunos de los grandes Expedientes de Regulación de Empleo llevados a cabo durante la crisis y de la reducción de puestos de trabajo como parte de los recortes de la Administración Pública, como el caso Musicop, que nace del despido de una treintena de trabajadores de la Escuela de Música de Mataró y de la oportunidad que surgió para los mismos la convocatoria de un concurso público municipal para gestionarla, que ganaron constituidos en cooperativa. ${ }^{18}$

Pero, como hemos apuntado, no todo son conflictos. En plena crisis, a veces los mismos dueños deciden transformar la empresa en cooperativa, algo que les ayuda a llevar mejor la recuperación, como en el caso de la valenciana Industrias Manclús, dedicada a la construcción de campanarios. Y también existen empresas que son transferidas a los trabajadores ante la falta de relevo generacional de empresarios que se jubilan y que deciden dejar la empresa a los trabajadores. ${ }^{19}$

Lo que sí parece claro es que cuando las iniciativas de recuperación surgen iniciado el procedimiento de concurso de acreedores no llegan a buen término. Esto es así porque en muchos casos, si hay activos valiosos en juego - como inmuebles o maquinaria - y se llega a la subasta, es muy probable que la gane un inversor externo en detrimento de los trabajadores, que no pueden igualar las ofertas de tales inversores. ${ }^{20}$

La Unión Europea, como hemos visto, está ya promoviendo la regulación de normas que fomenten estas prácticas por parte de los Estados miembros, pero lo cierto es que no existen regulaciones de este tipo, al menos en nuestro país, aunque sí normas puntuales que pueden ser interpre-

17 Información publicada por la Asociación de Empresas de Economía Social de Navarra (ANEL). Esta institución apunta, además, que dos de cada tres nuevos empleos que se crearon en Navarra en el sector privado son de empresas de Economía Social, según datos del segundo semestre de 2014.

18 Actualmente, gestionan varias escuelas de música y proyectos con ayuntamientos y centros culturales (http://www.musicop.cat/som-cooperativa.)

19 Es reseńable la experiencia pionera de las cooperativas de trabajo francesas, que reunidas en la federación Scop EnTREPRISES, crearon un proyecto junto al Ministerio de Industria, diseńado especialmente para traspasar empresas solventes, cuyos dueńos se jubilaban, como cooperativas.

20 Así sucedió en el caso de INOXgRUP, una empresa reconvertida que se dedicaba a fabricar los bolígrafos InOXCRom. Además, los antiguos empleados constituidos en cooperativa mantienen un litigio en los tribunales con los compradores por el uso de la marca (http://www.europapress.es/economia/noticia-economia-empresas-exempleados-inoxcrom-relanzan-marca-materialescritura-cataluna-madrid-20131023134223.html.) 
tadas de forma que favorezcan estos procesos. Nos referimos, por ejemplo, a la posibilidad de capitalizar la prestación por desempleo. ${ }^{21}$

\section{Aproximación a la fiscalidad de la transformación de la sociedad de capital en cooperativa}

\section{III.1. Aspectos fiscales reestructuración empresarial: precisión terminológica}

La expresión mercantil modificaciones estructurales hace referencia a un conjunto de operaciones a través de las cuales se produce un cambio en la estructura y en la organización de las sociedades que las llevan a cabo. Por tanto, el término comprende «aquellas alteraciones de la sociedad que van más allá de las simples modificaciones estatutarias para afectar a la estructura patrimonial o personal de la sociedad, y que, por tanto, incluyen la transformación, la fusión, la escisión y la cesión global de activo y pasivo.» ${ }^{22}$ No obstante, la sobradamente conocida autonomía del ordenamiento tributario a la hora de definir y configurar los institutos jurídicos sometidos a tributación, permite ampliar o reducir las definiciones o conceptos que de estos institutos dan otras leyes, para acomodarlos mejor a la exigencia de la tributación. ${ }^{23}$ Pues bien, en este caso, el concepto fiscal parece ser más restringido que el mercantil, puesto que entre las operaciones de reestructuración empresarial del capítulo VII del Título VII de la Ley 27/2014, de 27 de noviembre, del Impuesto sobre Sociedades (LIS), ${ }^{24}$ no incluye las operaciones de transformación societaria, por lo que no les resultará de aplicación este régimen especial, sino — como ha reconocido en distintas ocasiones la Dirección General de Tributos (DGT)—, ${ }^{25}$ se re-

${ }^{21}$ En este sentido, Argentina modificó, no sin cierta polémica, la Ley Concursal para dar preferencia las adquisiciones de las empresas por los trabajadores en el seno de los procedimientos concursales, otorgando a los créditos salariales (que son privilegiados) la cualidad de transformarse en capital para constituir la cooperativa en la que se convertirá la empresa en crisis.

22 Exposición de motivos de la LMESM.

23 Saínz de Bujanda, F. (1962): Hacienda y Derecho, t. I, Madrid, IEP, p. 41.

24 «Régimen especial de las fusiones, escisiones, aportaciones de activos, canje de valores y cambio de domicilio social de una Sociedad Europea o una Sociedad Cooperativa Europea de un Estado miembro a otro de la Unión Europea.» (coincidente con el Capítulo VIII del mismo título del Real Decreto Legislativo 4/2004, de 5 de marzo, por el que se aprueba el Texto Refundido de la Ley del Impuesto sobre Sociedades [TRLIS].)

25 V0855-05 (de 16/05/2005); V0795-06 (de 21/04/2006); V0297-09 (de 16/02/2009) o V2124-12 (de 06/11/2012), en contestación a consultas vinculantes relativas al TRLIS, por lo que señalan a aplicación del art. 26.2 TRLIS, equivalente del 27.2 de la LIS. 
girán por lo dispuesto en el art. 27.2 LIS, que regula los supuestos excepcionales de conclusión del período impositivo.

En el Derecho tributario español, la fusión y la escisión se configuran como fenómenos de naturaleza traslativa a título oneroso y otorga un régimen de neutralidad fiscal a estas operaciones de aplicación voluntaria por los sujetos pasivos que intervengan en ellas. Para Bahía Almansa, "quizás el legislador (en referencia al tributario específicamente) entendió que la transformación, al tener naturaleza meramente modificativa y no traslativa, no precisaba la aplicación de este régimen especial apoyado en la neutralidad.» ${ }^{26}$

\section{III.2. La transformación de la sociedad de capital en sociedad cooperativa en el Impuesto sobre Sociedades}

Aunque vamos a centrar el análisis en apuntar las consecuencias fiscales de la transformación societaria en un solo impuesto, que consideramos de mayor relevancia, hay un primer y evidente efecto fiscal de la transformación de una sociedad de capital, sea del tipo que sea, en una sociedad cooperativa que no despliega sus consecuencias exclusivamente en este impuesto: la necesidad de obtener un nuevo Número de Identificación Fiscal para realizar cualquier trámite con relevancia tributaria, lo que no se refiere exclusivamente a las relaciones entre la sociedad la $\mathrm{Ha}$ cienda Pública, sino que incluye todas las operaciones económicas que la sociedad realice. ${ }^{27}$

Además, en el momento en que la transformación sea efectiva, ${ }^{28}$ en aplicación del art. 27.2 LIS, se considerará concluido el período impositivo para la sociedad de capital transformada y comenzará un nuevo período impositivo para la cooperativa recién nacida a resultas del proceso de reestructuración. Según la letra d) de este precepto, concluirá en período impositivo "cuando se produzca la transformación de la forma so-

26 Bahía Almansa (2007): Análisis fiscal de la transformación de sociedades, Madrid, La Ley, p.71. El paréntesis es nuestro.

27 Esta cuestión está regulada en la Orden EHA/451/2008, de 20 de febrero, por la que se regula la composición del NIF de las personas jurídicas y entidades sin personalidad jurídica. Según esta norma, los NIF de las Sociedades Anónimas empiezan por la letra A y los de las Sociedades de Responsabilidad Limitada comienzan por la letra B. La nueva empresa está obligada a solicitar de la Agencia Tributaria un nuevo NIF mediante presentación del modelo 036 (Censo de empresarios, profesionales y retenedores-Declaración censal de alta, modificación y baja) para obtener un NIF que comience por la letra G.

${ }^{28}$ Esto es, en la fecha de la inscripción de la transformación en el Registro Mercantil. 
cietaria de la entidad, o la modificación de su estatuto o de su régimen jurídico, y ello determine la modificación de su tipo de gravamen o la aplicación de un régimen tributario distinto. La renta derivada de la transmisión posterior de los elementos patrimoniales existentes en el momento de la transformación o modificación, se entenderá generada de forma lineal, salvo prueba en contrario, durante todo el tiempo de tenencia del elemento transmitido. La parte de dicha renta generada hasta el momento de la transformación o modificación se gravará aplicando el tipo de gravamen y el régimen tributario que hubiera correspondido a la entidad de haber conservado su forma, estatuto o régimen originario.» ${ }^{29}$ El período impositivo no excederá de 12 meses [letra d) del mismo precepto.]

En este punto, para conocer el régimen tributario aplicable a la cooperativa resultante de la transformación, debemos atender a su carácter de cooperativa protegida, cooperativa especialmente protegida, o cooperativa no protegida. A cada una de estas categorías le resultará de aplicación unas u otras normas del régimen especial regulado en la Ley 20/1990, de 19 de diciembre, de Régimen Fiscal de Cooperativas (LRFC), para las cooperativas asentadas en CC.AA. de régimen común y el previsto en las correspondientes Leyes Forales para las cooperativas navarras y vascas ${ }^{30}$. A título de ejemplo, a las cooperativas que no gozan de protección fiscal, solo les resultaran de aplicación las normas de ajuste técnico y no las normas que establecen beneficios fiscales para las cooperativas $^{31}$.

En caso de reestructuraciones socialmente sostenibles, lo normal es que se constituya una cooperativa de trabajo asociado, tipología susceptible de ser considerada como cooperativa especialmente protegida. ${ }^{32} \mathrm{El}$ art. 8

$29 \mathrm{El}$ art. 27.2.d) LIS ha supuesto la mejora de la redacción del art. 26.2.d) TRLIS, de cuyo tenor literal se desprendía que en los supuestos de la letra d) el tipo de cambio que había de producirse en el régimen de tributación tenía que ser, siempre, del régimen general al especial o de uno especial a otro especial. Si se pasaba de un régimen especial a otro general sin cambio de tipo de gravamen - como ocurre en el supuesto de transformación de una sociedad mercantil en una cooperativa no protegida.-

30 Son la Ley Foral 9/1994, de 21 de junio, de Régimen Fiscal de Cooperativas de Navarra; Ley Foral 2/1997, de 22 de mayo, de Régimen Fiscal de Cooperativas de Guipúzcoa; Ley Foral 9/1997, de 14 de octubre, de Régimen Fiscal de Cooperativas de Vizcaya; y Ley Foral 16/1997, de 18 de junio, de Régimen Fiscal de Cooperativas de Álava.

31 Se considerarán cooperativas no protegidas fiscalmente aquéllas que no se constituyan con arreglo a los principios y disposiciones de la Ley sustantiva que les sea de aplicación o que incurran en alguna de las causas de pérdida de la condición de cooperativa fiscalmente protegida reguladas en el art. 13 LRFC.

32 Cfr. art. 7 LRFC. 
LRFC exige, para que gocen de esta consideración, que las cooperativas de trabajo asociado cumplan los siguientes requisitos ${ }^{33}$ :

1. Que asocien a personas físicas que presten su trabajo personal en la cooperativa para producir en común bienes y servicios para terceros.

2. ${ }^{\circ}$ Que el importe medio de sus retribuciones totales efectivamente devengadas, incluidos los anticipos y las cantidades exigibles en concepto de retornos cooperativos no excedan del $200 \%$ de la media de las retribuciones normales en el mismo sector de actividad, que hubieran debido percibir si su situación respecto a la cooperativa hubiera sido la de trabajadores por cuenta ajena.

3. ${ }^{\circ}$ Que el número de trabajadores asalariados con contrato por tiempo indefinido no exceda del $10 \%$ del total de sus socios. Sin embargo, si el número de socios es inferior a diez, podrá contratarse un trabajador asalariado. ${ }^{34} \mathrm{La}$ cooperativa podrá emplear trabajadores por cuenta ajena mediante cualquier otra forma de contratación, sin perder su condición de especialmente protegida, siempre que el número de jornadas legales realizadas por estos trabajadores durante el ejercicio económico no supere el 20 por 100 del total de jornadas legales de trabajo realizadas por los socios. Para el cómputo de estos porcentajes no se tomarán en consideración:

a) Los trabajadores con contrato de trabajo en prácticas, para la formación en el trabajo o bajo cualquier otra fórmula establecida para la inserción laboral de jóvenes.

b) Los socios en situación de suspensión o excedencia y los trabajadores que los sustituyan.

c) Aquellos trabajadores asalariados que una Cooperativa deba contratar por tiempo indefinido en cumplimiento de lo dispuesto en el artículo 44 dela Ley 8/1980, de 10 de marzo, del Estatuto de los Trabajadores, en los casos expresamente autorizados.

d) Los socios en situación de prueba.

33 El mismo precepto, en su apartado 4 dispone que «A efectos fiscales, se asimilarán a las cooperativas de trabajo asociado cualquier otra que, conforme a sus estatutos, adopte la forma de trabajo asociado, resultándose de aplicación las disposiciones correspondientes a esta clase de cooperativas.»

34 El cálculo de este porcentaje se realizará en función del número de socios y trabajadores asalariados existentes en la cooperativa durante el ejercicio económico, en proporción a su permanencia efectiva en la misma. 
El cumplimiento de estos requisitos tan exigentes, permitirá que la recién nacida cooperativa disfrute en el Impuesto sobre Sociedades de los beneficios fiscales establecidos para las cooperativas especialmente protegidas y que son, por un lado, los recogidos en los art. 33 (cooperativas protegidas) y art. 34 LRFC (cooperativas especialmente protegidas.) ${ }^{35}$

Los primeros, regulados en art. $33 \mathrm{LRFC}$, son:

1. La aplicación de un tipo reducido de gravamen del $20 \%$ a la base imponible, positiva o negativa, correspondiente a los resultados cooperativos (a la base imponible, positiva o negativa, correspondiente a los resultados extracooperativos se le aplicará el tipo general.) $)^{36}$

2. La libertad de amortización de los elementos de activo fijo nuevo amortizable, adquiridos en el plazo de tres años a partir de la fecha de su inscripción en el Registro de Cooperativas y Sociedades Anónimas Laborales del Ministerio de Trabajo y Seguridad Social, o en su caso, de las Comunidades Autónomas. ${ }^{37}$ Este beneficio es com-

$35 \mathrm{Al}$ estar estudiando exclusivamente las consecuencias fiscales de las reestructuraciones socialmente sostenibles en el Impuesto sobre Sociedades, dejamos a un lado los beneficios fiscales que también se reconocen para otros impuestos. En el Impuesto de Transmisiones Patrimoniales y Actos Jurídicos Documentados, exención respecto de: los actos de constitución, ampliación de capital, fusión y escisión; la constitución y cancelación de préstamos; las adquisiciones de bienes y derechos que se integren en el Fondo de Educación y Promoción para el cumplimiento de sus fines. Y para las especialmente protegidas, exención para las operaciones de adquisición de bienes y derechos destinados directamente al cumplimiento de sus fines sociales y estatutarios. En los tributos locales, gozarán de una bonificación del 95\% de la cuota, y en su caso de los recargos, en los siguientes tributos: Impuesto sobre Actividades Económicas; Impuesto sobre Bienes lnmuebles en los bienes de naturaleza rústica de las Cooperativas Agrarias y de Explotación Comunitaria de la Tierra.

36 En este punto no podemos obviar que desde 2006, ańo se produjo la primera revisión a la baja del tipo general del IS con efectos para el ejercicio 2007, venimos exigiendo que esa bajada se traslade también al tipo «bonificado» previsto para los resultados cooperativos de las cooperativas que gozan de protección fiscal. Y es inevitable porque el agravio comparativo se ha acrecentado aun más: la nueva LIS instaura, a partir del ejercicio 2016, un tipo general del 25\% (para el ejercicio 2015 la Disposición transitoria trigésima cuarta sobre medidas temporales aplicables en el período impositivo 2015 establece un tipo del 28\%) más un tipo del 15\% para las entidades de nueva creación en el primer periodo impositivo en que la base imponible resulte positiva y en el siguiente. Máxime si reparamos en que se ha utilizado la LIS para modificar el contenido de la LRFC en materia de compensación de pérdidas (véase nota 40) y se ha obviado esta reforma.

37 La cantidad fiscalmente deducible en concepto de libertad de amortización, una vez practicada la amortización normal de cada ejercicio en cuantía no inferior a la mínima, no podrá exceder del importe del saldo de la cuenta de resultados cooperativos disminuido en las aplicaciones obligatorias al Fondo de Reserva Obligatorio y participaciones del personal asalariado. 
patible, en su caso, para los mismos elementos, con la deducción por inversiones prevista en la LIS.

El beneficio fiscal específico para las cooperativas especialmente protegidas en este impuesto es una bonificación del 50\% de la cuota íntegra (art. 34 LRFC).

Para mantener la consideración de cooperativa protegida no se puede incurrir en una de las cusas que provocan la pérdida de tal condición previstas en el art 13 LRFC. ${ }^{38}$ El cumplimiento de estos requisi-

38 Estas se pueden sintetizar así:

1. Causas relativas a los fondos cooperativos: no hacer las dotaciones al FRO y al FEP en los supuestos, condiciones y cuantías previstas en las disposiciones cooperativas; repartir tanto el activo sobrante en el momento de la liquidación de la Cooperativa como los fondos que tengan carácter de irrepartibles entre los socios durante la vida de la sociedad; y aplicar a finalidades distintas de las previstas por la Ley, cantidades del FEP.

2. Causas relativas a los socios: acreditar a los socios en proporción distinta a las entregas, actividades o servicios realizados con la cooperativa o distribuirlos a terceros no socios; exceder de los límites legales autorizados las aportaciones al capital social de los socios; o tener un número de socios inferior al previsto en las normas legales, sin que se restablezca en un plazo de seis meses.

3. Causas producidas por incumplimiento de normas sustantivas: incumplir la normativa reguladora del destino del resultado de la regularización del balance de la cooperativa y del resultado de la actualización de las aportaciones de los socios al capital social; retribuir las aportaciones de los socios o asociados al capital social con intereses superiores a los máximos autorizados o superar tales límites en el abono de intereses por demora en el supuesto de reembolso de dichas aportaciones o por los retornos cooperativos devengados y no repartidos, por incorporarse a un Fondo Especial constituido por acuerdo de la Asamblea General; no imputar las pérdidas del ejercicio económico o imputarlas vulnerando las normas establecidas en la Ley, los Estatutos o los acuerdos de la Asamblea General; realizar más del 50\% de las operaciones cooperativizadas de la cooperativa con terceros no socios, fuera de los casos establecidos en la Ley; contratar trabajadores asalariados en número superior al que autoriza la ley; y la falta de auditoría externa en los casos señalados en las normas legales.

4. Otras causas: participar en el capital social de entidades no cooperativas en cuantía superior al 10\% (y más del 50\% del los recursos propios de la cooperativa). La cuantía podrá ascender al $40 \%$ si la participación se produce respecto de entidades que realicen actividades preparatorias, complementarias o subordinadas a las de la propia cooperativa. Además, la participación podrá ser superior si el Ministerio de Economía y Hacienda lo autoriza; incumplir las normas sobre contabilización separada de operaciones con terceros no socios: reducir el capital social a una cantidad inferior a la cifra mínima establecida estatutariamente, sin que se restablezca en un plazo de seis meses; paralizar la actividad cooperativizada la actividad los órganos sociales durante dos ańos, sin causa que lo justifique; y concluir la empresa que constituye su objeto o la imposibilidad manifiesta de desarrollar la actividad cooperativizada. 
tos afectará, por ejemplo, a la operatividad de la sociedad transformada con terceros no socios, que ahora se verá limitada; o si fuera matriz de un grupo, o participara en otras sociedades de capital en alguna medida, dicha participación se verá constreñida por los porcentajes establecidos en el art. 13.9. El momento de la transformación será también el momento de decidir la conveniencia de seguir actuando en el tráfico de la misma manera en que lo venían haciendo, asumiendo que no se podrán aplicar las normas de beneficio fiscal de la LRFC, o ajustarse a los requisitos de dicha Ley para aprovechar el régimen tributario especial.

En cualquier caso, en la transformación que analizamos de sociedad de capital a sociedad cooperativa, el cambio de forma de la entidad no origina que la sociedad creada deje de resultar gravada por el Impuesto sobre Sociedades, por lo que no habría que gravar las plusvalías latentes en los elementos patrimoniales al tiempo de la transformación. No obstante, según el art. 27.2.d) «La renta derivada de la transmisión posterior de los elementos patrimoniales existentes en el momento de la transformación o modificación, se entenderá generada de forma lineal, salvo prueba en contrario, durante todo el tiempo de tenencia del elemento transmitido. La parte de dicha renta generada hasta el momento de la transformación o modificación se gravará aplicando el tipo de gravamen y el régimen tributario que hubiera correspondido a la entidad de haber conservado su forma, estatuto o régimen originario.» Esta regla implica que la cooperativa resultante de la transformación tendrá que dividir la plusvalía generada por la transmisión de elementos patrimoniales existentes en el momento de la reestructuración, tomando como referencia la fecha de la transformación y gravando la parte generada anteriormente de acuerdo con el régimen tributario que le correspondía a la sociedad originaria y la parte generada tras la operación al régimen correspondiente a la sociedad cooperativa creada.

Junto a la conclusión del período impositivo por efecto de la transformación, se apunta la interesante cuestión de la compensación de pérdidas. La posibilidad de trasladar las bases imponibles negativas producidas en un ejercicio a los siguientes con bases imponibles positivas, para que sean compensadas, se regula con carácter general en el art. 26 LIS. Para conocer si tras una operación de transformación susbsiste este beneficio, debemos averiguar qué factor otorga a la sociedad la facultad de compensar pérdidas fiscalmente reconocidas en un ejercicio con otros posteriores con beneficios: ya sea la permanencia de la sociedad como sujeto de derecho; ya sea la permanencia de los socios que hayan sufrido la pérdida; ya sea la permanencia de la actividad económica que ha producido la misma. En el sistema tributario español parece primar la continuidad en el tiempo de la cualidad 
de sujeto pasivo del impuesto. ${ }^{39}$ Esta interpretación se refuerza atendiendo a que el principio esencial de la transformación societaria es que la personalidad jurídica de la entidad transformada se conserva. ${ }^{40}$

Así entendido el asunto, en el caso que nos ocupa, no se plantean problemas al respecto puesto que tanto la entidad originaria como la resultante son sujetos pasivos del Impuesto sobre Sociedades, si bien no debe escapársenos que, en la mayoría de los casos - esto es, en tanto la cooperativa nacida del proceso goce de protección fiscal-, el régimen tributario aplicable a las mismas será distinto, lo que conlleva un cambio a la hora de realizar la compensación. La regulación que hace el art. 24.1 LRFC sustituirá, en este supuesto, a la que contiene el art. 26 LIS como consecuencia de la diferente tributación de los resultados cooperativos y extracooperativos (así lo establece expresamente el apartado 2 del mismo precepto). Por tanto, «si la suma algebraica a que se refiere el artículo anterior resultase negativa, su importe podrá compensarse por la cooperativa con las cuotas íntegras positivas de los períodos impositivos siguientes, con el límite del 60\% de la cuota íntegra previa a su compensación. En todo caso, serán compensables en el período impositivo cuotas íntegras por el importe que resulte de multiplicar 1 millón de euros al tipo medio de gravamen de la entidad.» ${ }^{41}$

39 Bahía Almansa (2007, p. 224).

40 Cfr. art. 3 LMESM: «En virtud de la transformación una sociedad adopta un tipo social distinto, conservando su personalidad jurídica.»

${ }^{41}$ Redacción dada por el apartado uno del apartado tercero de la disposición final cuarta de la Ley 27/2014, de 27 de noviembre, del Impuesto sobre Sociedades, con efectos para los períodos impositivos que se inicien a partir de 1 de enero de 2015.

Cfr. disposición transitoria octava sobre compensación de cuotas negativas en el año 2015, redactada por la misma Ley: «El límite a que se refiere el apartado 1 del artículo 24 de esta Ley no resultará de aplicación en los períodos impositivos que se inicien en el año 2015.

No obstante, la compensación de cuotas negativas de ejercicios anteriores, para los contribuyentes cuyo volumen de operaciones, calculado conforme a lo dispuesto en el artículo 121 de la Ley 37/1992, de 28 de diciembre, del Impuesto sobre el Valor Añadido, haya superado la cantidad de 6.010.121,04 euros durante los 12 meses anteriores a la fecha en que se inicien los períodos impositivos dentro del año 2015, tendrá los siguientes límites:

- La compensación de cuotas negativas está limitada al 50\% de la cuota íntegra previa a dicha compensación, cuando en esos 12 meses el importe neto de la cifra de negocios sea al menos de 20 millones de euros pero inferior a 60 millones de euros.

- La compensación de cuotas negativas está limitada al 25\% de la cuota íntegra previa a dicha compensación, cuando en esos 12 meses el importe neto de la cifra de negocios sea al menos de 60 millones de euros.

La limitación a la compensación de cuotas negativas no resultará de aplicación en el importe de las rentas correspondientes a quitas y esperas consecuencia de un acuerdo con los acreedores no vinculados con el contribuyente.» 
Como puede verse, se deroga el plazo de diez ejercicios para compensar contenido en la anterior regulación. ${ }^{42}$

Cabría preguntarse aquí, así entendida la continuidad subjetiva entre las empresas participantes en la operación de reestructuración, si es posible acogerse al tipo de gravamen del $15 \%$ para las entidades de nueva creación en el primer período impositivo en que la base imponible resulte positiva y en el siguiente. En nuestra opinión, los supuestos en que no se entenderá iniciada una actividad económica — regulados en el art. 29.1 letras a) y b) - no parece que puedan ajustarse a todas las situaciones que originan una reestructuración socialmente sostenible. Por un lado, la cooperativa resultante de la transformación no será una entidad vinculada en el sentido del art. 18 LIS [letra a)]. Y por otro, solo podrá considerarse que la actividad económica ha sido ejercida, durante el año anterior a la constitución de la entidad por una persona física que ostente una participación, directa o indirecta, en el capital o en los fondos propios de la entidad de nueva creación superior al 50\% [letra b)], en el caso en que sea el propio empresario, propietario de una SL, el que propicie la creación de la cooperativa con sus trabajadores y se quede con un porcentaje de participación superior al 50\%. Pero esta situación sería excepcional porque tal porcentaje, en una cooperativa, no le da ventaja respecto a otros cooperativistas en relación al derecho de voto y, por tanto, al control de la entidad; ni en cuanto a los derechos de retorno, que es como se llaman los beneficios que perciben los socios de las cooperativas. En ningún caso podría llegar a ese porcentaje como socio inversor. En consecuencia, si no se dan los supuestos en los que no se entiende iniciada la actividad empresarial, en sentido contrario, podríamos considerar que la cooperativa nacida de la transformación es una entidad de nueva creación a los efectos de poder aplicarse el tipo gravamen previsto para esta situación. En contra de esta interpretación juega la conservación de la personalidad jurídica de la sociedad transformada, si bien no podemos olvidar que la función de la transformación es evitar pasos intermedios para llegar a la misma finalidad a la que llegaríamos por la disolución de una sociedad y creación de una nueva.

42 Este plazo solo se contempla ya para limitar el derecho de la Administración para comprobar o investigar las cuotas negativas pendientes de compensación, a contar desde el día siguiente a aquel en que finalice el plazo establecido para presentar la declaración o autoliquidación correspondiente al período impositivo en que se generó el derecho a su compensación. Transcurrido dicho plazo, el contribuyente deberá acreditar que las cuotas negativas cuya compensación pretenda resultan procedentes, así como su cuantía, mediante la exhibición de la liquidación o autoliquidación y de la contabilidad, con acreditación de su depósito durante el citado plazo en el Registro Mercantil (art. 24.1 in fine). 
Una última cuestión que es necesario al menos dejar apuntada es la que atañe a la conversión de las bonificaciones fiscales por reinversión de los beneficios de las sociedades en una reserva de capitalización, ${ }^{43}$ que impediría disfrutar de los mismos a la cooperativa resultante del proceso de transformación societaria puesto que, tal y como está configurada en el art. 25 LIS, solo resulta aplicable a «los contribuyentes que tributen al tipo de gravamen previsto en los apartados 1 o 6 del artículo 29 de esta Ley tendrán derecho a una reducción en la base imponible del 10 por ciento del importe del incremento de sus fondos propios.» ${ }^{44}$ Esto limitaría la posibilidad de aplicar el beneficio contenido en este precepto exclusivamente a las cooperativas que no gocen de protección fiscal, que serán las que tributen al tipo general, puesto que las que gocen protección tendrían que fragmentar su base imponible a fin de aplicar los tipos correspondientes a los resultados cooperativos y extracooperativos. Se podría discutir aquí, si por la parte relativa a estos últimos, que se gravan al tipo general, habría posibilidad de aplicar la reserva de capitalización. No creemos que sea ésta la voluntad de legislador por como ha redactado el precepto y por las dificultades técnicas que ello entrañaría.

\section{Notas conclusivas}

Muy a menudo, el problema al que se enfrentan las transmisiones empresariales a los empleados en forma de cooperativa no solo radica en la duración de los procedimientos en cuestión, sino también, y lo que es más importante, en la falta de conocimiento de este contexto empresarial por parte de los profesionales interesados (abogados y contables) y dentro del

43 La LIS elimina las deducciones por reinversión de beneficios extraordinarios y por inversión de beneficios creada recientemente, sustituyendo ambos incentivos por uno nuevo denominado reserva de capitalización que, según la Exposición de Motivos de la misma, «se traduce en la no tributación de aquella parte del beneficio que se destine a la constitución de una reserva indisponible, sin que se establezca requisito de inversión alguno de esta reserva en algún tipo concreto de activo. Con esta medida se pretende potenciar la capitalización empresarial mediante el incremento del patrimonio neto, $y$, con ello, incentivar el saneamiento de las empresas y su competitividad. Asimismo, esta medida conjuntamente con la limitación de gastos financieros neutraliza en mayor medida el tratamiento que tiene en el Impuesto sobre Sociedades la financiación ajena frente a la financiación propia, objetivo primordial tras la crisis económica y en consonancia con las recomendaciones de los organismos internacionales» [II.4 letra b)].

${ }^{44}$ Se refiere a aquellas entidades que tributan al tipo general del $25 \%$ (apartado 1 ) y al tipo del 30\% (apartado 6). 
sistema jurídico y académico. Por tanto, la investigación en este ámbito, para poner herramientas a disposición de todos los actores que intervienen en la creación o la transmisión de empresas a los empleados contribuirían en gran medida a fomentar esta práctica.

Pero la realidad nos dice también que esto no basta: para que estas reestructuraciones socialmente sostenibles se lleven a cabo con éxito deben apoyarse financieramente por el sector público, algo que ya han defendido algunas instituciones de la Unión Europea, tanto mediante la creación de una partida presupuestaria específica como mediante un uso más preciso e inteligente de los Fondos Estructurales. Resulta asimismo indispensable que la Comisión Europea que establezca dispositivos para facilitar la cooperación y el intercambio de mejores prácticas y métodos entre las empresas e inste a los Estados miembros a regular normas que favorezcan estas operaciones.

El análisis fiscal, por su parte, refleja que la nueva Ley del Impuesto sobre Sociedades no ha cerrado los interrogantes que ya se planteaban con la legislación anterior, obligando a la doctrina a una nueva revisión del estado de la cuestión para clarificar las cuestiones controvertidas. Se ha perdido una gran oportunidad para resolver dudas y para coordinar la legislación fiscal y la mercantil en este campo. ${ }^{45}$

\section{Bibliografía}

Álvarez Royo-Villanova, S. y González-Meneses, M. (2013): Modificaciones estructurales de las sociedades mercantiles, Dykinson, Madrid.

Bahía Almansa, B. (2007): Análisis fiscal de la transformación de sociedades, La Ley,Madrid,.

BirchaLl, J. (2014): Resilience of the cooperative business model in times of crisis, OIT, Ginebra.

Calvo Ortega, R. (2005): Fiscalidad de las entidades de economía social: cooperativas, mutuas, sociedades laborales, fundaciones, asociaciones de utilidad püblica, centros especiales de empleo, empresas de inserción social, Civitas, Madrid.

CECOP-CICOPA Europe: Business Transfers to Employees under the Form of a Cooperative in Europe. Opportunities and Challenges, junio, 2013, disponible http://www.cecop.coop/IMG/pdf/bussinesstransferstoemployeesundertheformofacooperativeineuropececop-4.pdf

45 Álvarez Royo-Villanova y González-Meneses advierten en su obra Modificaciones estructurales de las sociedades mercantiles (Madrid, Dykinson, 2013, p. 23) que la LMESM «deja subsistente la tradicional dicotomía o incluso esquizofrenia de nuestro derecho de modificaciones estructurales, dividido en dos regulaciones paralelas: la normativa mercantil sustantiva y la norma fiscal (...) que no son del todo coincidentes en cuanto a la delimitación y tipificación de los supuestos». Ello provoca disfunciones que no se han resuelto con la Ley actual. 
Comisión Europea COM(2005) 120 final: Reestructuraciones y empleo-Anticipar y acompañar las reestructuraciones para desarrollar el empleo: el papel de la Unión Europea, 31 de marzo de 2005.

Comité Económico y Social Europeo (CCMI/093-CESE1049-2012): Dictamen sobre el tema "Cooperativas y reestructuración", 25 de abril de 2012.

Divar Garteiz-Aurrecoa, J. (2013): «Las cooperativas: Una alternativa económica y social frente a la crisis", Boletín de la Asociación Internacional de Derecho Cooperativo, núm. 47/2013, pp. 257-264.

Parlamento Europeo (2012/2321(INI)): Informe sobre la contribución de las cooperativas a la salida de la crisis, 12 de junio de 2013.

SaÍnz de Bujanda, F. (1962): Hacienda y Derecho, t. I, IEP, Madrid.

VARgas VAsserot, C. et al. (2015): Derecho de las sociedades cooperativas: Introducción, constitución, estatuto del socio y órganos sociales, La Ley, Madrid.

Vilnitzky, M. y Ruiz, O. (2010): «Dossier Empresas recuperadas», El Viejo topo, núm. 270-271, julio-agosto 2010, pp. 21 a 34. 